\title{
A preemptive repeat priority queue with resampling: performance analysis
}

Joris Walraevens*, Bart Steyaert and Herwig Bruneel ${ }^{\dagger}$

\section{SMACS Research Group}

Department of Telecommunications and Information Processing (TW07)

Ghent University - UGent

Sint-Pietersnieuwstraat 41, B-9000 Gent, Belgium.

Phone: +32-9-2648902, fax: +32-9-2644295

E-mail: \{jw,bs,hb\}@telin.UGent.be

${ }^{\dagger}$ This work has been supported by the Interuniversity Attraction Poles Programme - Belgian Science Policy. 


\begin{abstract}
In this paper, we analyze a discrete-time preemptive repeat priority queue with resampling. High-priority packets have preemptive repeat priority, and interrupted low-priority packets are subjected to independent retransmission attempts. Both classes contain packets with generally distributed transmission times. We show that the use of generating functions is beneficial for analyzing the system contents and packet delay of both classes. The influence of the priority scheduling on the performance measures is illustrated by some numerical examples.
\end{abstract}

Keywords - discrete-time queueing theory, priority scheduling 
Buffers with a priority scheduling discipline are widely studied in the literature. The results obtained for these queueing systems, can be used when several types of packets (or traffic, jobs, customers, ...) share the same resources, but some are more delay-sensitive than others. In this paper, we will focus on the effect of a specific priority scheme, i.e., the preemptive repeat priority scheduling discipline with resampling. We assume that delay-sensitive traffic has priority over delay-insensitive traffic, i.e., when the server becomes free, a packet of delay-sensitive traffic, when available, will always be scheduled next. In the remaining, we will refer to the delay-sensitive and delay-insensitive traffic as high- and low-priority traffic respectively. Newly arriving high-priority traffic interrupts the transmission of a low-priority packet that has already commenced, and the interrupted low-priority packet has to restart its service afterwards. Its new service time upon retransmission is not necessarily the same as the old one, but it takes a new sample (with an identical distribution).

In the literature, there have been a number of contributions with respect to priority scheduling (see a.o. Jaiswal (1968)). Specifically, queues with a preemptive priority repeat scheduling discipline have been analyzed in Sumita and Sheng (1988); Yoon and Un (1994); Mukherjee Saha and Tripathi (1995); Hong and Takagi (1997); Fiems Steyaert and Bruneel (2003). In Sumita and Sheng (1988), a database system with update requests and read queries is analyzed, where the former have preemptive repeat priority over the latter. Yoon and Un (1994) describe the use of preemptive priority repeat scheduling in CSMA-CD protocols for fiber optic bus networks. These papers stress that preemptive repeat priority queues are widely applicable in different fields of computer science, telecommunication networks and production processes. Mukherjee Saha and Tripathi (1995) study a preemptive repeat with resampling scheduling of voice traffic over data traffic in a ring-based LAN. Hong and Takagi (1997) analyze the transmission delay in a priority-based packet-switching system with three phases of preemption mechanism, namely a preemptive repeat, a preemptive resume and a non-preemptive phase. Finally, Fiems Steyaert and Bruneel (2003) analyze queueing models with service interruptions, which can be applied (in some cases) to analyze priority queues.

In this paper, we analyze the system contents and packet delay of high-priority and low-priority traffic in a discrete-time single-server buffer with a preemptive repeat with resampling priority scheme and per-slot i.i.d. numbers of arrivals. The transmission times of the packets are assumed to be generally distributed. These distributions are class-dependent, i.e., the transmission times of the high-priority packets can be different from those of the low-priority packets (which reflects the case where different classes represent different applications). We will demonstrate that an analysis based on generating functions is extremely suitable for modeling this type of buffers with a priority scheduling discipline and for calculating the relevant performance measures.

The remainder of this paper is structured as follows. In the following section, we present the mathematical model. In sections 2 and 3, we analyze the steady-state system contents and packet delay of both classes. We discuss the obtained results in section 4 and some numerical examples are treated in section 5. Finally, some conclusions are formulated in section 6. 


\section{Definitions and mathematical model}

We consider a discrete-time single-server system with infinite buffer space. Time is assumed to be slotted. There are two types of packets arriving to the system, namely packets of class-1 and packets of class- 2 . The numbers of arrivals of class- $j$ during slot $k$ are i.i.d. and are denoted by $a_{j, k}(j=1,2)$. The joint probability generating function (pgf) of $a_{1, k}$ and $a_{2, k}$ is defined as

$$
A\left(z_{1}, z_{2}\right) \triangleq \mathrm{E}\left[z_{1}^{a_{1, k}} z_{2}^{a_{2, k}}\right]
$$

The marginal pgfs of the number of per-slot arrivals of class- $j$ are denoted by $A_{j}(z)(j=1,2)$ and are given by $A(z, 1)$ and $A(1, z)$ respectively. We will furthermore denote the mean arrival rate of class- $j$ packets by $\lambda_{j} \triangleq \mathrm{E}\left[a_{j, k}\right]=A_{j}^{\prime}(1)(j=1,2)$.

The service times of the class- $j$ packets, i.e., the numbers of slots it takes to transmit complete class- $j$ packets, are i.i.d. and generally distributed and their pgf is denoted by $S_{j}(z)(j=1,2)$. The mean service time of a class- $j$ packet is denoted by $\mu_{j}=S_{j}^{\prime}(1)(j=1,2)$. For further use, we define the total arrival load as $\rho_{T}=\lambda_{1} \mu_{1}+\lambda_{2} \mu_{2}$.

The class-1 packets are assumed to have preemptive repeat with resampling priority over the class- 2 packets and within one class the scheduling is FCFS.

\section{System contents}

We denote the system contents of class- $j$ packets at the beginning of slot $k$ by $u_{j, k}(j=1,2)$. Since the service times of both classes are generally distributed, the set $\left\{u_{1, k}, u_{2, k}\right\}$ does not form a Markov chain. Therefore, we introduce an additional stochastic variable $r_{k}$ as follows: $r_{k}$ indicates the residual service time, i.e., the remaining number of slots needed to transmit the packet in service, at the beginning of slot $k$, if $u_{T, k}>0$, and $r_{k}=0$ if $u_{T, k}=0 . u_{T, k} \triangleq u_{1, k}+u_{2, k}$ denotes the total system contents at the beginning of slot $k$. With these definitions, $\left\{r_{k}, u_{1, k}, u_{2, k}\right\}$ is seen to constitute a Markovian state description of the system at the beginning of slot $k$. If $s_{j, k}^{*}(j=1,2)$ indicates the service time of the next class- $j$ packet to receive service after slot $k$, the following system equations can be established:

1. If $r_{k}=0$ (and hence $\left.u_{T, k}=0\right)$ :

$$
u_{1, k+1}=a_{1, k} ; u_{2, k+1}=a_{2, k} ; r_{k+1}= \begin{cases}0 & \text { if } u_{1, k+1}=u_{2, k+1}=0 \\ s_{2, k}^{*} & \text { if } u_{1, k+1}=0, u_{2, k+1}>0 \\ s_{1, k}^{*} & \text { if } u_{1, k+1}>0\end{cases}
$$

The system was empty at the beginning of slot $k$. If no packets arrive during slot $k$, the system stays empty. If no class-1 packets and at least one class-2 packet arrives during slot $k$, a class-2 packet starts 
receiving service at the beginning of slot $k+1$. If at least one class- 1 packet arrives during slot $k$, a class-1 packet enters the service unit.

2. If $r_{k}=1$ :

(a) If $u_{1, k}=0$ :

$$
u_{1, k+1}=a_{1, k} ; u_{2, k+1}=u_{2, k}-1+a_{2, k} ; r_{k+1}= \begin{cases}0 & \text { if } u_{1, k+1}=u_{2, k+1}=0 \\ s_{2, k}^{*} & \text { if } u_{1, k+1}=0, u_{2, k+1}>0 \\ s_{1, k}^{*} & \text { if } u_{1, k+1}>0\end{cases}
$$

A class-2 packet left the system at the end of slot $k$. A new packet (of class-1, if any, otherwise of class-2) enters the service unit at the beginning of slot $k+1$ (if the system is non-empty at that time instant).

(b) If $u_{1, k}>0$ :

(4) $u_{1, k+1}=u_{1, k}-1+a_{1, k} ; u_{2, k+1}=u_{2, k}+a_{2, k} ; r_{k+1}=\left\{\begin{array}{cl}0 & \text { if } u_{1, k+1}=u_{2, k+1}=0 \\ s_{2, k}^{*} & \text { if } u_{1, k+1}=0, u_{2, k+1}>0 \\ s_{1, k}^{*} & \text { if } u_{1, k+1}>0\end{array}\right.$.

A class-1 packet left the system at the end of slot $k$. A new packet commences service during slot $k+1$.

3. If $r_{k}>1$ :

(a) If $u_{1, k}=0$ :

$$
u_{1, k+1}=a_{1, k} ; u_{2, k+1}=u_{2, k}+a_{2, k} ; r_{k+1}= \begin{cases}r_{k}-1 & \text { if } u_{1, k+1}=0 \\ s_{1, k}^{*} & \text { if } u_{1, k+1}>0\end{cases}
$$

A class-2 packet was in service during slot $k$, but needs at least one more slot to complete its service. The class-2 packet stays in the server when no new class-1 packets arrived during slot $k$, otherwise its service is preempted by one of the newly arrived class- 1 packets (because of the preemptive priority scheduling).

(b) If $u_{1, k}>0$ :

$$
u_{1, k+1}=u_{1, k}+a_{1, k} ; u_{2, k+1}=u_{2, k}+a_{2, k} ; r_{k+1}=r_{k}-1 \text {. }
$$

A class-1 packet was in service during slot $k$, but needs at least one more slot to complete its service. 
Now, let us define $P_{k}\left(x, z_{1}, z_{2}\right) \triangleq \mathrm{E}\left[x^{r_{k}} z_{1}^{u_{1, k}} z_{2}^{u_{2, k}}\right]$ as the joint pgf of the state vector $\left(r_{k}, u_{1, k}, u_{2, k}\right)$. In the remainder, we define $\mathrm{E}[X\{Y\}]$ as $\mathrm{E}[X \mid Y] \operatorname{Prob}[Y]$. Using the system equations, we can constitute a relation between $P_{k}\left(x, z_{1}, z_{2}\right)$ and $P_{k+1}\left(x, z_{1}, z_{2}\right)$. We assume that the system is stable (we will comment on the stability condition later) and as a result $P_{k}\left(x, z_{1}, z_{2}\right)$ and $P_{k+1}\left(x, z_{1}, z_{2}\right)$ converge both to a common steadystate value $P\left(x, z_{1}, z_{2}\right)=\lim _{k \rightarrow \infty} P_{k}\left(x, z_{1}, z_{2}\right)$. By taking the $k \rightarrow \infty$ limit in the relation between $P_{k}\left(x_{1}, z_{1}, z_{2}\right)$ and $P_{k+1}\left(x_{1}, z_{1}, z_{2}\right)$, we obtain:

$$
\begin{aligned}
P\left(x, z_{1}, z_{2}\right)= & \frac{1}{x-A\left(z_{1}, z_{2}\right)}\left\{\left[x A(0,0)\left(1-S_{2}(x)\right)+A\left(0, z_{2}\right)\left(x S_{2}(x)-1\right)\right] P(0,0,0)\right. \\
& +x A(0,0)\left(1-S_{2}\left(x_{2}\right)\right)\left[R_{1}(0,0)+R_{2}(0)\right]+x A\left(0, z_{2}\right)\left(S_{2}(x)-S_{1}(x)\right) R_{1}\left(0, z_{2}\right) \\
& +x\left[A\left(0, z_{2}\right)\left(S_{2}(x)-z_{2}\right)+\left(A\left(z_{1}, z_{2}\right)-A\left(0, z_{2}\right)\right) S_{1}(x)\left(1-z_{2}\right)\right] R_{2}\left(z_{2}\right) \\
& \left.+\left(A\left(z_{1}, z_{2}\right)-A\left(0, z_{2}\right)\right)\left[x S_{1}(x) P\left(1,0, z_{2}\right)-P\left(x, 0, z_{2}\right)\right]+x A\left(z_{1}, z_{2}\right)\left(S_{1}(x)-z_{1}\right) R_{1}\left(z_{1}, z_{2}\right)\right\},
\end{aligned}
$$

with $R_{1}\left(z_{1}, z_{2}\right) \triangleq \lim _{k \rightarrow \infty} \mathrm{E}\left[z_{1}^{u_{1, k}-1} z_{2}^{u_{2, k}}\left\{r_{k}=1, u_{1, k}>0\right\}\right]$ and $R_{2}\left(z_{2}\right) \triangleq \lim _{k \rightarrow \infty} \mathrm{E}\left[z_{2}^{u_{2, k}-1}\left\{r_{k}=1, u_{1, k}=0\right\}\right]$. It now remains for us to determine the unknown functions $P\left(x, 0, z_{2}\right), R_{1}\left(z_{1}, z_{2}\right)$ and $R_{2}\left(z_{2}\right)$. This can be done in a few steps. Firstly, we observe that $P(x, 0,0)=P(0,0,0)$, due to the fact that $r_{k}=0$ if and only if $u_{1, k}=u_{2, k}=0$. By putting $z_{j}=0(j=1,2)$ in (7) and using this observation, we obtain:

$$
P(0,0,0)=A(0,0)\left[P(0,0,0)+R_{1}(0,0)+R_{2}(0)\right] .
$$

Replacing $z_{1}$ by 0 in equation (7) and using equation (8), we find the following expression for $P\left(x, 0, z_{2}\right)$ :

$$
\begin{aligned}
P\left(x, 0, z_{2}\right)= & \frac{1}{x-A\left(0, z_{2}\right)}\left\{\left[x\left(1-S_{2}(x)\right)+A\left(0, z_{2}\right)\left(x S_{2}(x)-1\right)\right] P(0,0,0)\right. \\
& \left.+x A\left(0, z_{2}\right) S_{2}(x) R_{1}\left(0, z_{2}\right)+x A\left(0, z_{2}\right)\left(S_{2}(x)-z_{2}\right) R_{2}\left(z_{2}\right)\right\}
\end{aligned}
$$

We note that $P\left(x, 0, z_{2}\right)$ is bound for all values of $x$ and $z_{2}$ such that $|x| \leq 1$ and $\left|z_{2}\right| \leq 1$ since $P\left(x, z_{1}, z_{2}\right)$ is a pgf. In particular, this should be true for $x=A\left(0, z_{2}\right),\left|z_{2}\right| \leq 1$, since $\left|A\left(0, z_{2}\right)\right| \leq 1$ for all such $z_{2}$. If we choose $x=A\left(0, z_{2}\right)$ in equation (9), where $\left|z_{2}\right| \leq 1$, the denominator in the right hand side of this equation equals zero. The above then implies that the numerator in the right hand side of equation (9) also equals zero, which yields the following expression:

$$
R_{1}\left(0, z_{2}\right)=\frac{S_{2}\left(A\left(0, z_{2}\right)\right)\left(1-A\left(0, z_{2}\right)\right) P(0,0,0)+A\left(0, z_{2}\right)\left(z_{2}-S_{2}\left(A\left(0, z_{2}\right)\right)\right) R_{2}\left(z_{2}\right)}{A\left(0, z_{2}\right) S_{2}\left(A\left(0, z_{2}\right)\right)} .
$$

Going back to expression (7), we notice that $P\left(x, z_{1}, z_{2}\right)$ must be bound for all values of $x$ and $z_{j}$ such that $|x| \leq 1$ and $\left|z_{j}\right| \leq 1(j=1,2)$ since $P\left(x, z_{1}, z_{2}\right)$ is a pgf. In particular, this should be true for $x=A\left(z_{1}, z_{2}\right)$, $\left|z_{j}\right| \leq 1(j=1,2)$, since $\left|A\left(z_{1}, z_{2}\right)\right| \leq 1$ for all such $z_{j}$. Substituting $x$ by $A\left(z_{1}, z_{2}\right)$ in equation (7), where 
$\left|z_{j}\right| \leq 1$, the denominator in the right hand side of this equation vanishes. The same must then be true for the numerator of the right hand side of equation (7), which yields the following expression (by taking into account the expressions of $P\left(x, 0, z_{2}\right)$ and $R_{1}\left(0, z_{2}\right)$ obtained in equations (9) and (10) respectively):

$$
\begin{aligned}
& R_{1}\left(z_{1}, z_{2}\right) \\
& \quad \frac{S_{1}\left(A\left(z_{1}, z_{2}\right)\right)}{\left(1-A\left(0, z_{2}\right)\right) A\left(z_{1}, z_{2}\right) S_{2}\left(A\left(0, z_{2}\right)\right)\left(z_{1}-S_{1}\left(A\left(z_{1}, z_{2}\right)\right)\right)}\left\{( 1 - A ( 0 , z _ { 2 } ) ) ( A ( z _ { 1 } , z _ { 2 } ) - 1 ) S _ { 2 } \left(A\left(0, z_{2}\right) P(0,0,0)\right.\right. \\
& \text { (11) } \left.\quad+\left[\left(A\left(z_{1}, z_{2}\right)-A\left(0, z_{2}\right)\right) S_{2}\left(A\left(0, z_{2}\right)\right)\left(1-z_{2}\right)+A\left(0, z_{2}\right)\left(A\left(z_{1}, z_{2}\right)-1\right)\left(z_{2}-S_{2}\left(A\left(0, z_{2}\right)\right)\right)\right] R_{2}\left(z_{2}\right)\right\} .
\end{aligned}
$$

Once again, we note that $\left(1-A\left(0, z_{2}\right)\right) A\left(z_{1}, z_{2}\right) S_{2}\left(A\left(0, z_{2}\right)\right) R_{1}\left(z_{1}, z_{2}\right)$ must be bound for all values of $z_{j}$ such that $\left|z_{j}\right| \leq 1(j=1,2)$. In particular, this should be true for $z_{1}=Y\left(z_{2}\right)$, with $Y(z) \triangleq S_{1}(A(Y(z), z))$, and $\left|z_{2}\right| \leq 1$, since it follows from Rouché's theorem that there is exactly one solution $\left|Y\left(z_{2}\right)\right| \leq 1$ for all such $z_{2}$. We point out that $Y(1)$ equals 1 . The above implies that if we insert $z_{1}=Y\left(z_{2}\right)$ in equation (11), where $\left|z_{2}\right| \leq 1$, the denominator in the right hand side of this equation vanishes. The same must then be true for the numerator in the right hand side, yielding

$$
R_{2}\left(z_{2}\right)=P(0,0,0) \frac{\left(A\left(Y\left(z_{2}\right), z_{2}\right)-1\right) B\left(z_{2}\right)}{A\left(Y\left(z_{2}\right), z_{2}\right)\left(z_{2}-B\left(z_{2}\right)\right)},
$$

with

$$
B(z)=\frac{(1-A(0, z)) A(Y(z), z) S_{2}(A(0, z))}{(A(Y(z), z)-A(0, z)) S_{2}(A(0, z))-A(0, z)(A(Y(z), z)-1)} .
$$

An almost fully determined version for $P\left(x, z_{1}, z_{2}\right)$ can now be derived by substituting the equations (8)-(12) in $(7)$ :

$$
\begin{aligned}
P\left(x, z_{1}, z_{2}\right)= & P(0,0,0)\left\{1+x z_{1} \frac{\left(A\left(z_{1}, z_{2}\right)-A\left(Y\left(z_{2}\right), z_{2}\right)\right)\left(S_{1}(x)-S_{1}\left(A\left(z_{1}, z_{2}\right)\right)\right) B\left(z_{2}\right)\left(z_{2}-1\right)}{A\left(Y\left(z_{2}\right), z_{2}\right)\left(x-A\left(z_{1}, z_{2}\right)\right)\left(z_{1}-S_{1}\left(A\left(z_{1}, z_{2}\right)\right)\right)\left(z_{2}-B\left(z_{2}\right)\right)}\right. \\
& \left.+x z_{2} \frac{A\left(0, z_{2}\right)\left(A\left(Y\left(z_{2}\right), z_{2}\right)-1\right)\left(S_{2}(x)-S_{2}\left(A\left(0, z_{2}\right)\right)\right) B\left(z_{2}\right)}{A\left(Y\left(z_{2}\right), z_{2}\right) S_{2}\left(A\left(0, z_{2}\right)\right)\left(x-A\left(0, z_{2}\right)\right)\left(z_{2}-B\left(z_{2}\right)\right)}\right\} .
\end{aligned}
$$

Finally, in order to find an expression for $P(0,0,0)$, we put $x=z_{1}=z_{2}=1$ in equation (14) and use de l'Hôpital's rule. We obtain

$$
P(0,0,0)=1-\rho_{T, e f f},
$$

with $\rho_{T, e f f}=\rho_{1}+\lambda_{2} \mu_{2, e f f}$ and $\mu_{2, e f f}=\frac{A_{1}(0)\left(1-S_{2}\left(A_{1}(0)\right)\right)}{S_{2}\left(A_{1}(0)\right)\left(1-A_{1}(0)\right)}$. Using this result in equation (14), we finally obtain a fully determined, albeit elaborate expression for $P\left(x, z_{1}, z_{2}\right)$. From this pgf, we can calculate the 
joint pgf of the system contents of class- 1 and class- 2 packets. It is given by:

$$
\begin{aligned}
U\left(z_{1}, z_{2}\right) \triangleq & \lim _{k \rightarrow \infty} \mathrm{E}\left[z_{1}^{u_{1, k}} z_{2}^{u_{2, k}}\right]=P\left(1, z_{1}, z_{2}\right) \\
= & \left(1-\rho_{T, e f f}\right)\left\{1+z_{1} \frac{\left(A\left(z_{1}, z_{2}\right)-A\left(Y\left(z_{2}\right), z_{2}\right)\right)\left(1-S_{1}\left(A\left(z_{1}, z_{2}\right)\right)\right) B\left(z_{2}\right)\left(z_{2}-1\right)}{\left.A\left(z_{2}\right), z_{2}\right)\left(1-A\left(z_{1}, z_{2}\right)\right)\left(z_{1}-S_{1}\left(A\left(z_{1}, z_{2}\right)\right)\right)\left(z_{2}-B\left(z_{2}\right)\right)}\right. \\
& \left.+z_{2} \frac{A\left(0, z_{2}\right)\left(A\left(Y\left(z_{2}\right), z_{2}\right)-1\right)\left(1-S_{2}\left(A\left(0, z_{2}\right)\right) B\left(z_{2}\right)\right.}{A\left(Y\left(z_{2}\right), z_{2}\right) S_{2}\left(A\left(0, z_{2}\right)\right)\left(1-A\left(0, z_{2}\right)\right)\left(z_{2}-B\left(z_{2}\right)\right)}\right\} .
\end{aligned}
$$

From the two-dimensional pgf $U\left(z_{1}, z_{2}\right)$, we derive the expressions for the marginal pgfs of the system contents of class-1 and class-2 packets respectively at the beginning of an arbitrary slot, yielding

$$
\begin{aligned}
& U_{1}(z) \triangleq \lim _{k \rightarrow \infty} \mathrm{E}\left[z^{u_{1, k}}\right]=U(z, 1)=\left(1-\rho_{1}\right) \frac{S_{1}\left(A_{1}(z)\right)(z-1)}{z-S_{1}\left(A_{1}(z)\right)} \\
& U_{2}(z) \triangleq \lim _{k \rightarrow \infty} \mathrm{E}\left[z^{u_{2, k}}\right]=U(1, z)=\left(1-\rho_{T, e f f}\right) \frac{A_{2}(z)(1-A(Y(z), z))}{A(Y(z), z)\left(1-A_{2}(z)\right)} \frac{B(z)(z-1)}{z-B(z)} .
\end{aligned}
$$

By taking the necessary derivatives of these marginal pgfs, moments of the class- 1 and class- 2 system contents can be found. The mean class- 2 system contents are for instance given by

$$
\begin{aligned}
E\left[u_{2}\right]= & U_{2}^{\prime}(1) \\
= & \rho_{2, \text { eff }}+\frac{\lambda_{2}\left(\mu_{2, e f f}-1\right) \rho_{T, e f f}}{1-\rho_{T, e f f}}-\frac{A^{(2)}(0,1) S_{2}^{\prime}\left(A_{1}(0)\right) \rho_{2, e f f}}{S_{2}\left(A_{1}(0)\right)\left(1-S_{2}\left(A_{1}(0)\right)\right)\left(1-\rho_{T, e f f}\right)} \\
& +\frac{A^{(2)}(0,1) \rho_{2, e f f}}{A_{1}(0)\left(1-A_{1}(0)\right)\left(1-\rho_{T, \text { eff }]}\right)}+\frac{\lambda_{2}\left(\lambda_{1} \mu_{11}+\lambda_{11} \mu_{1}^{2}\right)}{2\left(1-\rho_{1}\right)\left(1-\rho_{T, e f f}\right)}+\frac{2 \lambda_{12} \mu_{1}+\lambda_{22} \mu_{2, \text { eff }}}{2\left(1-\rho_{T, \text { eff }}\right)} .
\end{aligned}
$$

Higher moments of the system contents can also be calculated straight-forwardly from expressions (17)-(18), but the expressions are too elaborate to show.

\section{Delay}

The packet delay is defined as the number of slots between the end of a random packet's arrival slot and the end of its departure slot. Due to the preemptive priority scheduling, we can analyze the packet delay of class-1 packets as if they are the only packets in the system. This is already done e.g. in Bruneel and Kim (1993).

Deriving an expression for $D_{2}(z)$, the pgf of a class-2 packet delay (denoted by $d_{2}$ ) will be a bit more involved. We tag a class- 2 packet that enters the buffer during slot $k$. Let us refer to the packets in the system at the end of slot $k$, but that have to be served before the tagged packet as the "primary packets". So, basically, the tagged class-2 packet can enter the server, when all primary packets and all class-1 packets that arrived after slot $k$ are transmitted. In order to analyze the delay of the tagged class- 2 packet, only the number of class- 1 and class- 2 packets that are served between the arrival slot of the tagged class-2 packet 
and its departure slot is important, not the precise order in which they are served. Therefore, in order to facilitate the analysis, we will consider an equivalent virtual system with an altered service discipline. We assume that from slot $k+1$ on, the order of service for class-1 packets (those in the queue at the end of slot $k$ and newly arriving ones) is LCFS instead of FCFS in the equivalent system (the transmission of class-2 packets remains FCFS). So, a primary packet can enter the server, when the system becomes free (for the first time) of class-1 packets that arrived during and after the service time of the primary packet that predecessed it according to the new service discipline. Let $v_{j, m}^{(i)}$ denote the length of the time period during which the server is occupied by the $m$-th class- $j$ packet that arrives during slot $i$ and its class- 1 "successors", i.e., the time period starting at the beginning of the service of that packet and terminating when the system becomes free (for the first time) of that packet and class-1 packets which arrived during and after its service time. The $v_{j, m}^{(i)}$ 's $(j=1,2)$ are called sub-busy periods, initiated by the $m$-th class- $j$ packet that arrived during slot $i$. The sub-busy period started by the tagged packet itself is denoted by $\tilde{v}_{2}$.

During the tagged packet's arrival slot, the system is in one of the following states:

1. $u_{1, k}=u_{2, k}=0$ :

$$
d_{2}=\sum_{j=1}^{2} \sum_{m=1}^{f_{j, k}} v_{j, m}^{(k)}+\tilde{v}_{2}-\sum_{m=1}^{a_{1, k+d_{2}}} v_{1, m}^{\left(k+d_{2}\right)}
$$

with $f_{j, k}$ the number of class- $j$ packets arriving during slot $k$, but that have to be served before the tagged packet. $f_{j, k}$ class- $j$ primary packets $(j=1,2)$ and their class- 1 successors have to be served before the tagged class- 2 packet. During the service time of the tagged class- 2 packet, new class- 1 packets may arrive, which interrupt the tagged packet's service. The sub-busy periods initiated by the class-1 packets arriving in the slot preceding the departure of the tagged packet (slot $k+d_{2}$ ), is a part of $\tilde{v}_{2}$, but is not a part of the packet delay of the tagged packet, which accounts for the last term in the right hand side of the above expression.

2. $u_{1, k}=0, u_{2, k}>0$ :

$$
d_{2}=v_{2, k}^{+}+\sum_{m=1}^{u_{2, k}-1} \tilde{v}_{2, m}+\sum_{m=1}^{f_{2, k}} v_{2, m}^{(k)}+\tilde{v}_{2}-\sum_{m=1}^{a_{1, k+d_{2}}} v_{1, m}^{\left(k+d_{2}\right)}
$$

with $v_{2, k}^{+}$the remaining part of the sub-busy period initiated by the class-2 packet in service during slot $k$. The $\tilde{v}_{2, m}$ 's are defined as the sub-busy periods, caused by the $m$-th class- 2 packet already in the queue at the beginning of slot $k$. The difference with the former case, is that (multiple) class-2 packets are present in the system when the tagged class-2 packet arrives and thus have to be served before the tagged packet. All these class-2 packets initiate their own sub-busy periods.

3. $u_{1, k}>0$ :

$$
d_{2}=\left(r_{k}-1\right)+\sum_{i=1}^{r_{k}-1} \sum_{m=1}^{a_{1, k+i}} v_{1, m}^{(k+i)}+\sum_{j=1}^{2} \sum_{m=1}^{f_{j, k}} v_{j, m}^{(k)}+\sum_{m=1}^{u_{1, k}-1} \tilde{v}_{1, m}+\sum_{m=1}^{u_{2, k}} \tilde{v}_{2, m}+\tilde{v}_{2}-\sum_{m=1}^{a_{1, k+d_{2}}} v_{1, m}^{\left(k+d_{2}\right)}
$$


with the $\tilde{v}_{j, m}$ 's defined as the sub-busy periods, caused by the $m$-th class- $j$ packet already in the queue at the beginning of slot $k$. The residual service time of the class-1 packet in service during slot $k$ contributes in the first term, the sub-busy periods of the class-1 packets arriving during the residual service time contribute in the second term, the sub-busy periods of the class-1 and class- 2 packets arriving during slot $k$, but that have to be served before the tagged class-2 packet contribute in the third term, the sub-busy periods of the class- 1 and class- 2 packets already in the queue at the beginning of slot $k$ contribute in the fourth and the fifth term respectively and finally the service time of the tagged class-2 packet itself and the sub-busy periods of the class-1 packets arriving during this service time (except for its last slot) contribute in the last two terms.

It can easily be seen that the sub-busy periods initiated by the primary packets of class-1 (class- 2 respectively) form a set of i.i.d. random variables and their (common) pgf will be represented by $V_{1}(z)\left(V_{2}(z)\right.$ respectively). We will first concentrate on finding expressions for $V_{1}(z)$ and $V_{2}(z)$. $V_{1}(z)$ satisfies $V_{1}(z)=S_{1}\left(z A_{1}\left(V_{1}(z)\right)\right)$. This can be understood as follows: the sub-busy period initiated by a class-1 packet consists of two parts: the service time of that packet itself, and the sub-busy periods initiated by the class- 1 packets that arrive during its service time. This leads to the above relation for $V_{1}(z)$. The calculation of $V_{2}(z)$ is more complicated because of the preemptive repeat with resampling priority scheduling. When a class-2 packet enters the server, two events can occur: the class-2 packet can either be completely served in the first attempt, or the service of the class- 2 packet is interrupted by newly arriving class-1 packets. If interrupted before completing its service time, the low-priority packet has to wait in the queue until all class-1 packets are served and can then have another transmission attempt. Since the service time in the new attempt is resampled, this again initiates a new class-2 sub-busy period with same pgf $V_{2}(z)$. Transforming all this into the $z$-domain, we can find the following expression for $V_{2}(z)$ (after some extensive mathematical manipulations)

$$
V_{2}(z)=\frac{A_{1}\left(V_{1}(z)\right) S_{2}\left(A_{1}(0) z\right)\left(A_{1}(0) z-1\right)}{A_{1}(0)\left(z A_{1}\left(V_{1}(z)\right)-1\right)+S_{2}\left(A_{1}(0) z\right)\left(A_{1}\left(V_{1}(z)\right)-A_{1}(0)\right)}
$$

Taking the $z$-transform of equations (20)-(22), we find the following expression for $D_{2}(z)$ (after some mathematical manipulations)

$$
\begin{aligned}
D_{2}(z)= & \frac{V_{2}(z)}{A_{1}\left(V_{1}(z)\right)}\left\{F\left(V_{1}(z), V_{2}(z)\right)\left[P(0,0,0)+R_{2}\left(V_{2}(z)\right)\right]\right. \\
& +\left[F\left(V_{1}(z), V_{2}(z)\right)-F\left(0, V_{2}(z)\right) \frac{z A_{1}\left(V_{1}(z)\right)-1}{A_{1}(0) z-1}\right]\left[P\left(1,0, V_{2}(z)\right)-V_{2}(z) R_{2}\left(V_{2}(z)\right)-P(0,0,0)\right] \\
& +F\left(0, V_{2}(z)\right) \frac{z A_{1}\left(V_{1}(z)\right)-1}{A_{1}(0) z-1} \frac{P\left(A_{1}(0) z, 0, V_{2}(z)\right)-A_{1}(0) z V_{2}(z) R_{2}\left(V_{2}(z)\right)-P(0,0,0)}{A_{1}(0) z S_{2}\left(A_{1}(0) z\right)} \\
& \left.+F\left(V_{1}(z), V_{2}(z)\right) \frac{P\left(z A_{1}\left(V_{1}(z)\right), V_{1}(z), V_{2}(z)\right)-P\left(z A_{1}\left(V_{1}(z)\right), 0, V_{2}(z)\right)}{z A_{1}\left(V_{1}(z)\right) V_{1}(z)}\right\}
\end{aligned}
$$


with $P\left(x, z_{1}, z_{2}\right)$ and $R_{2}\left(z_{2}\right)$ defined in the previous section and

$$
F\left(z_{1}, z_{2}\right) \triangleq \mathrm{E}\left[z_{1}^{f_{1, k}} z_{2}^{f_{2, k}}\right]=\frac{A\left(z_{1}, z_{2}\right)-A_{1}\left(V_{1}(z)\right)}{\lambda_{2}\left(V_{2}(z)-1\right)}
$$

which can be found using the extension of a technique used in e.g. Bruneel and Kim (1993). Using expressions (12), (14) and (25) in the previous expression of $D_{2}(z)$, we finally find

$$
\begin{aligned}
D_{2}(z)= & \frac{1-\rho_{T, \text { eff }}}{\lambda_{2}} \frac{V_{2}(z)}{A_{1}\left(V_{1}(z)\right)} \frac{B\left(V_{2}(z)\right)}{A\left(Y\left(V_{2}(z)\right), V_{2}(z)\right)\left(V_{2}(z)-B\left(V_{2}(z)\right)\right)} \\
(26) \quad & \left\{\frac{\left(A\left(V_{1}(z), V_{2}(z)\right)-A_{1}\left(V_{1}(z)\right)\right)\left(z A_{1}\left(V_{1}(z)\right)-A\left(Y\left(V_{2}(z)\right), V_{2}(z)\right)\right)}{z A_{1}\left(V_{1}(z)\right)-A\left(V_{1}(z), V_{2}(z)\right)}+\frac{V_{2}(z)\left(A\left(0, V_{2}(z)\right)-A_{1}(0)\right)}{V_{2}(z)-1}\right. \\
& \left.\frac{\left(z A_{1}\left(V_{1}(z)\right)-1\right)\left(A\left(Y\left(V_{2}(z)\right), V_{2}(z)\right)-1\right)\left(C\left(A_{1}(0) z, A\left(0, V_{2}(z)\right)\right)-C\left(A\left(0, V_{2}(z)\right), A_{1}(0) z\right)\right)}{S_{2}\left(A_{1}(0) z\right) S_{2}\left(A\left(0, V_{2}(z)\right)\right)\left(A_{1}(0) z-1\right)\left(A\left(0, V_{2}(z)-1\right)\right)\left(A_{1}(0) z-A\left(0, V_{2}(z)\right)\right)}\right\},
\end{aligned}
$$

with $B(z)$ given by $(13)$ and $C(x, y)=x\left(S_{2}(x)-1\right)\left(y-S_{2}(y)\right)$. From this expression, moments of the packet delay can be calculated as for the system contents. We find e.g. $\mathrm{E}\left[d_{2}\right]=\mathrm{E}\left[u_{2}\right] / \lambda_{2}$ (with $\mathrm{E}\left[u_{2}\right]$ given by $(19)$ ), for the mean packet delay of a class-2 packet, as expected by the discretized version of Little's law (see Fiems and Bruneel (2002)).

\section{Discussion of results}

\subsection{Stability issues}

The studied queueing system is not work-conserving, since parts of class-2 packets have to be retransmitted. This is also apparent in the stability boundary of the queueing system. For the system to reach steady-state, the probability of an empty system in steady-state has to be strictly positive. The probability of an empty system is given by $P(0,0,0)$. From expression (15) it is seen that this probability is positive if $\rho_{T, \text { eff }}<1$. $\rho_{T, \text { eff }}$ is thus the effective load (including retransmission of class-2 packets) offered to the system and is for this particular queueing model - not identical to the arrival load $\rho_{T}=\lambda_{1} \mu_{1}+\lambda_{2} \mu_{2}$.

\subsection{Class-1 system contents}

The expression $(17)$ of $U_{1}(z)$ is equal to the generating function of the system contents in a single class FIFO queue with arrival and service process equal to this of class-1. This was expected since the low-priority packets have no influence on the high-priority packets and thus, from the high-priority packets point of view, they are the only packets in the system. 


\subsection{Special case: geometric class-2 service times}

If we assume geometric service times for the low-priority class, we obtain the same pgfs for $U\left(z_{1}, z_{2}\right)$ and $D_{2}(z)$ as found in Walraevens Steyaert and Bruneel (2005), where a buffer with a preemptive resume priority scheduling - with generally (geometrically respectively) distributed high-priority (low-priority respectively) service times - is analyzed. The difference between a preemptive resume (analyzed in Walraevens Steyaert and Bruneel (2005)) and a preemptive repeat (analyzed in this paper) priority scheduling discipline is that in the former an interrupted low-priority packet only has to transmit the not-yet-transmitted part after the interruption, while in the latter an interrupted packet has to be completely retransmitted. Since the geometric distribution is memoryless however, a residual service time and a complete service time have the same (geometric) distribution, and thus in this case the system contents in a buffer with preemptive resume priority on the one hand and preemptive repeat priority with resampling on the other hand are identically distributed.

\subsection{Special case: uncorrelated arrival process}

In the special case that the numbers of per-slot arrivals of class- 1 and class- 2 packets are uncorrelated, i.e. $A\left(z_{1}, z_{2}\right)=A_{1}\left(z_{1}\right) A_{2}\left(z_{2}\right)$, we can analyze the system contents of class-2 packets in an alternative way. Since class-2 packets can only be served when there are no class-1 packets in the system, we can model the system, with respect to class- 2 packets, in terms of a system with server interruptions. The server is blocked for class- 2 packets if there are class-1 packets waiting to be sent, and it is available if there are none. We can then calculate the pgf of the duration of busy and idle periods of class- 1 packets, i.e., the time period during which there are class- 1 packets in the system (i.e., $\left.u_{1}>0\right)$ and the time period during which there are no such packets (i.e., $u_{1}=0$ ), respectively. It is easily verified that the duration of the idle period is geometrically distributed, i.e., its pgf is given by

$$
P_{I}(z)=\frac{\left(1-A_{1}(0)\right) z}{1-A_{1}(0) z}
$$

The calculation of the busy period is a bit more involved, and can be found in Bruneel and Kim (1993):

$$
P_{B}(z)=\frac{A_{1}\left(S_{1}\left(z\left[\left(1-A_{1}(0)\right) P_{B}(z)+A_{1}(0)\right]\right)\right)-A_{1}(0)}{1-A_{1}(0)} .
$$

Note that the lengths of consecutive busy and idle periods are statistically independent. It is clear that when the system is busy with respect to class- 1 packets, it is blocked for class-2 packets. Therefore, with respect to class-2 packets, the system can be modeled as a single-server buffer with server interruptions, for which the lengths of consecutive available and blocked periods are i.i.d. and their respective pgfs are given by equations (27) and (28) and where the service time of interrupted packets have to be repeated with resampling. Such 
a queueing system has already been analyzed in Fiems and Bruneel (2003). Translating the results from this analysis to our case, the pgf of the system contents of class-2 packets becomes

$$
U_{2}(z)=\left(1-\rho_{T, \text { eff }}\right) \frac{1-A_{1}(X(z)) A_{2}(z)}{A_{1}(X(z))\left(1-A_{2}(z)\right)} \frac{B(z)(z-1)}{z-B(z)},
$$

with

$$
B(z)=\frac{\left(1-A_{1}(0) A_{2}(z)\right) A_{1}(X(z)) S_{2}\left(A_{1}(0) A_{2}(z)\right)}{\left(A_{1}(X(z))-A_{1}(0)\right) S_{2}\left(A_{1}(0) A_{2}(z)\right)-A_{1}(0)\left(A_{1}(X(z)) A_{2}(z)-1\right)},
$$

and

$$
X(z)=S_{1}\left(A_{1}(X(z)) A_{2}(z)\right)
$$

Equations (18) and (29) ((13) and (30) respectively) lead to the same result for $U_{2}(z)(B(z)$ respectively), when $X(z)=Y(z)$. This is the case when the number of class-1 and class-2 arrivals during a slot are uncorrelated.

Furthermore, equation (26) for $D_{2}(z)$ simplifies to

$$
D_{2}(z)=\frac{1-\rho_{T, \text { eff }}}{\lambda_{2}} \frac{\left(1-A_{2}\left(V_{2}(z)\right)\right) V_{2}(z)\left(1-z A_{1}\left(V_{1}(z)\right)\right)}{\left(A_{2}\left(V_{2}(z)\right)-z\right)\left(1-V_{2}(z)\right) A_{1}\left(V_{1}(z)\right)}
$$

for $A\left(z_{1}, z_{2}\right)=A_{1}\left(z_{1}\right) A_{2}\left(z_{2}\right)$. The same result can be found in Fiems and Bruneel (2003).

\subsection{Stochastic interpretation of $Y(z)$ and $B(z)$}

The function $Y(z)$ is the pgf of the stochastic variable $y$, which can be defined as the number of low-priority packets that arrive during a sub-busy period caused by a high-priority packet in the alternative service discipline, described in section 3 . If at the beginning of slot $k$ a high-priority packet with service time $\tilde{s}_{1}^{(k)}$ enters the server, a new sub-busy period starts. If we denote the number of class-2 packets that arrive during this sub-busy period by $\tilde{y}^{(k)}$, then

$$
\tilde{y}^{(k)}=\sum_{i=0}^{\tilde{s}_{1}^{(k)}-1}\left(a_{2, k+i}+\sum_{m=1}^{a_{1, k+i}} y_{m}^{(k+i)}\right)
$$

with $y_{m}^{(k+i)}$ the number of class-2 packets that arrive during the sub-busy period started by the $m$-th class 1 packet that arrives during slot $k+i$. Naturally, all $y_{m}^{(k+i)}$ have the same distribution as $\tilde{y}^{(k)}$ (since the lengths of all sub-busy periods are i.i.d.) and their pgf is thus indeed given by $Y(z)=S_{1}(A(Y(z), z))$, as immediately follows from (33) assuming that a stationary regime is reached.

The function $B(z)$ is the pgf of $b$, defined as the number of low-priority packets that arrive during a sub- 
busy period caused by a low-priority packet in the alternative service discipline, or - assuming a low-priority packet enters the server at the beginning of slot $k$ -

$$
b^{(k)}=\sum_{i=0}^{v_{2}^{(k)}-1} a_{2, k+i}
$$

with $v_{2}^{(k)}$ and $b^{(k)}$ the sub-busy period initiated by the low-priority packet entering the server at the beginning of slot $k$ and the number of class-2 packets arriving in the system during that sub-busy period respectively. Following a same reasoning as for the calculation of $V_{2}(z)$ in section 3, we indeed find expression (13) for the pgf of $b^{(k)}$ (in steady-state).

Note that $Y(z)$ and $B(z)$ are equal to $V_{1}\left(A_{2}(z)\right)$ and $V_{2}\left(A_{2}(z)\right)$ respectively when the number of per-slot arrivals of class- 1 and class- 2 are uncorrelated. In that case the length of the sub-busy period and the number of class-2 packets arriving during that sub-busy period are uncorrelated, because the length of the sub-busy period only depends on the number of class-1 arrivals during the sub-busy period. If the number of per-slot arrivals of class- 1 and class-2 packets are correlated on the other hand, the length of the sub-busy period and the number of class-2 arrivals during the sub-busy period are correlated: they both depend on the number of class-1 arrivals during the sub-busy period, thus not leading to such a straight-forward relation between $Y(z)\left(B(z)\right.$ respectively) and $V_{1}(z)\left(V_{2}(z)\right.$ respectively).

\section{Numerical examples}

In this section, we present a few numerical examples. We assume the traffic of the two classes to be arriving according to a two-dimensional binomial process. Its two-dimensional pgf is given by

$$
A\left(z_{1}, z_{2}\right)=\left(1-\frac{\lambda_{1}}{N}\left(1-z_{1}\right)-\frac{\lambda_{2}}{N}\left(1-z_{2}\right)\right)^{N}
$$

The arrival rate of class- $j$ traffic is thus given by $\lambda_{j}(j=1,2)$ and $N$ is assumed to be 16 . We assume deterministic service times for class-1 packets and we assume that class-2 packet lengths can take two possible values, 2 and 10 slots respectively, both with probability $1 / 2 . \alpha$ is defined as the fraction of class- 1 arrival load in the total arrival load.

In Figure 1, the mean value and variance of the system contents of class-1 and class-2 packets are shown as functions of the total arrival load, when the service times of class-1 are equal to 6 , and when $\alpha$ is 0.25 , 0.5 and 0.75 respectively. We see the influence of the priority scheduling discipline: the mean and variance of the high-priority system contents stay low, while the mean and variance of the low-priority contents are high (even for high $\alpha$, i.e., for many high-priority packets in the traffic mix).

[Figure 1 about here.] 
In Figure 2a., the mean packet delay of class-1 and class-2 packets is shown as a function of the total arrival load, when service times of class- 1 are equal to 6 , and when $\alpha$ is $0.25,0.5$ and 0.75 respectively. The influence of priority scheduling on the packet delay becomes obvious from this figure: mean delay of class-1 packets remains small while the price to pay is of course a larger mean delay of class-2 packets. Also, the smaller the fraction of high-priority packets in the overall traffic mix, the lower the mean packet delay of both classes will be. Figure 2b. shows the mean class-2 packet delay as a function of the class- 1 service time $\mu_{1}$, when the total arrival load is 0.75 and for $\alpha$ equal to $0.25,0.5$ and 0.75 . Two counter-acting effects can be observed: firstly, longer class-1 packets increase the build-up periods for class-2 packets in the queue (i.e., longer periods when the server is busy with class- 1 packets), thereby increasing the mean class-2 packet delay. Secondly, longer class-1 packets (while keeping the class-1 arrival load constant) means less class-1 packet arrivals, thus decreasing the probability of a class-2 packet's service getting preempted and having to retransmit. This has a decreasing effect on the mean class-2 packet delay. The latter effect is however only important for small class-1 service times and low class-1 arrival loads (as can be seen in the figure).

[Figure 2 about here.]

\section{Conclusion}

In this paper, we have analyzed a discrete-time queue with a preemptive repeat priority scheduling discipline with resampling and two priority classes. We have derived the joint probability generating function of the system contents of both classes and the probability generating functions of the packet delay of both classes. Performance measures can be calculated from these pgfs. We have shown the impact of the preemptive priority scheduling with resampling on the performance characteristics by some numerical examples. 


\section{References}

Bruneel, H., and B.G. Kim. (1993). Discrete-time models for communication systems including ATM, Boston: Kluwer Academic Publishers.

Fiems, D., and H. Bruneel. (2002). "A note on the discretization of Little's result" Operations Research Letters $30(1), 17-18$.

Fiems, D., B. Steyaert and H. Bruneel. (2003). "Analysis of a discrete-time GI-G-1 queueing model subjected to bursty interruptions," Computers and Operations Research 30(1), 139-153.

Fiems, D. and H. Bruneel. (2003)."Discrete-time queues with correlated vacations", In Proceedings of the Eighteenth International Teletraffic Congress, ITC 18 (Berlin, 31 August - 5 September 2003), 581-590.

Hong, S.J., and H. Takagi. (1997). "Analysis of transmission delay for a structured-priority packet-switching system," Computer Networks and ISDN Systems 29(6), 701-715.

Jaiswal, N. K. (1968). Priority Queues, Academic Press.

Mukherjee, S., D. Saha and S.K. Tripathi. (1995). "A preemptive protocol for voice-data intergration in ring-based LAN: performance analysis and comparison," Performance Evaluation 23(1), 1-29.

Sumita, U., and O.R.L. Sheng. (1988). "Analysis of query processing in distributed database systems with fully replicated files: a hierarchical approach," Performance Evaluation 8(3), 223-238.

Yoon, C.H., and C.K. Un. (1994). "Unslotted 1-persistent and $p_{i}$-persistent CSMA-CD Protocols for fiber optic bus networks," IEEE Transactions on Communications 42(2-4), 458-465.

Walraevens, J., B. Steyaert and H. Bruneel. (2003). "Performance analysis of a single-server ATM queue with a priority scheduling," Computers and Operations Research 30(12), 1807-1829.

Walraevens, J., B. Steyaert and H. Bruneel. (2005). "A packet switch with a priority scheduling discipline: performance analysis," Telecommunication Systems 28(1): 53-77. 


\section{List of Figures}

1 Moments of system contents versus the total arrival load f . . . . . . . . . . . . . 18

2 Mean packet delay . . . . . . . . . . . . . . . . . . . . . . . . . 19 


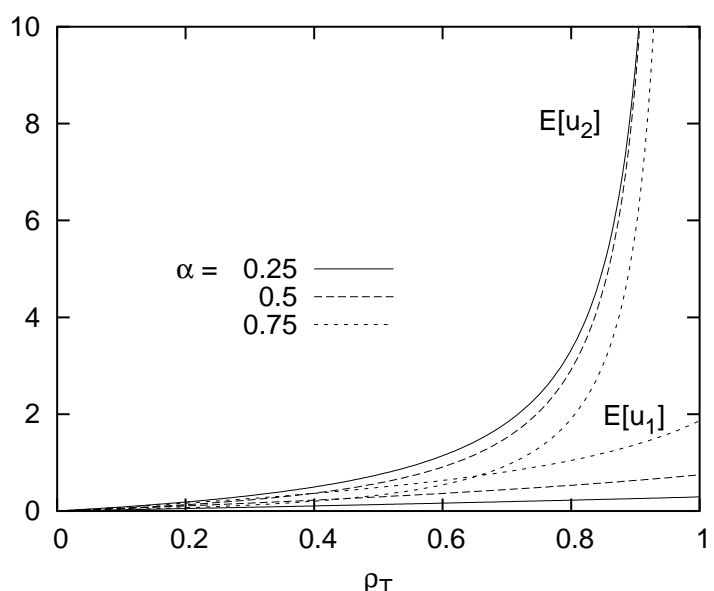

a. Mean

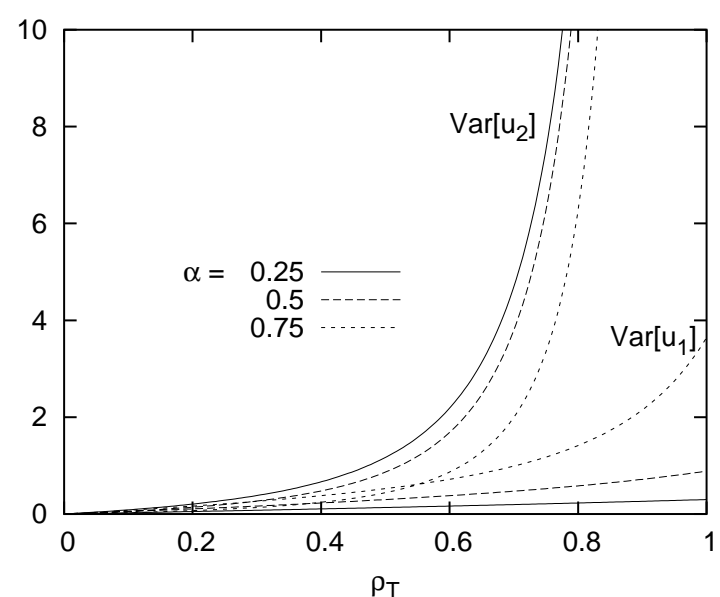

b. Variance

Figure 1: Moments of system contents versus the total arrival load 


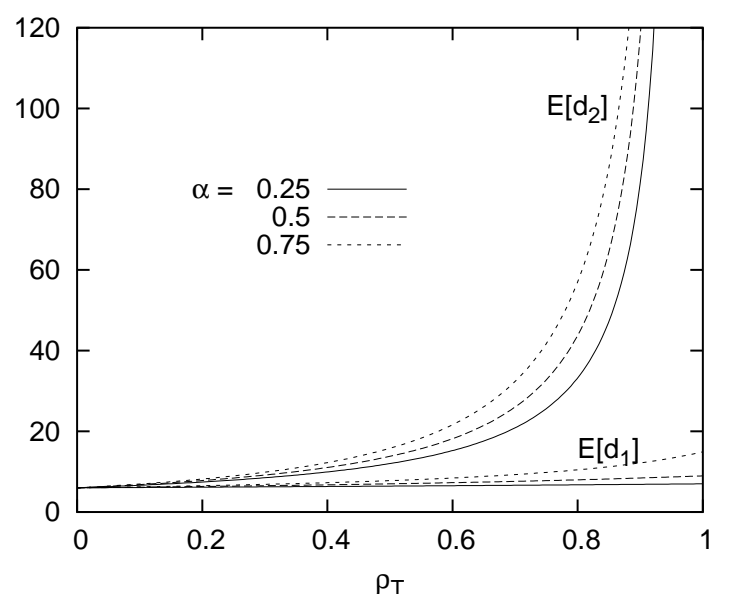

a. Mean packet delay versus the total arrival load

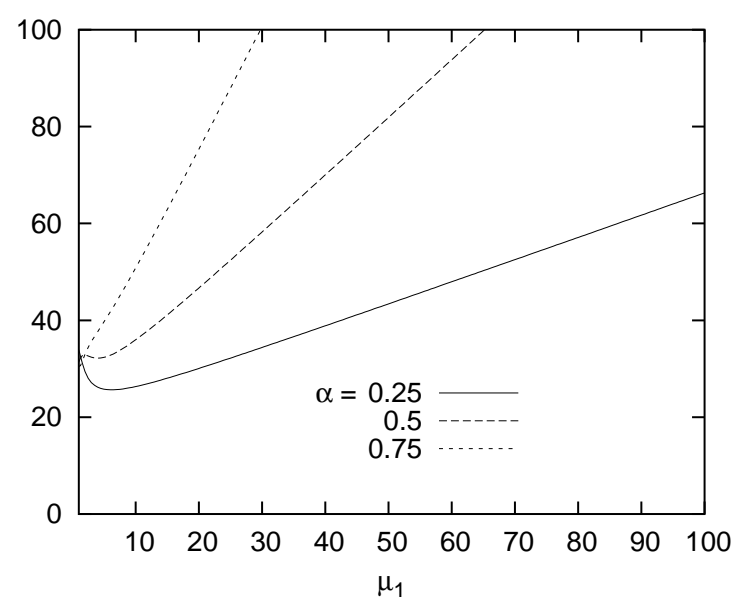

b. Mean class-2 packet delay versus the class-1 service time

Figure 2: Mean packet delay 